\title{
Unconditionally Explicit Stable Difference Schemes for Solving Some Linear and Non-Linear Parabolic Differential Equation
}

\author{
Masaharu Nakashima \\ Kagoshima-shi, Taniyama Chuou 1-4328 891-0141, Japan \\ Email: m_naka304@yahoo.co.jp
}

Received 18 September 2015; accepted 24 November 2015; published 27 November 2015

Copyright (C) 2015 by author and Scientific Research Publishing Inc.

This work is licensed under the Creative Commons Attribution International License (CC BY).

http://creativecommons.org/licenses/by/4.0/

(c) (i) Open Access

\begin{abstract}
We present the numerical method for solution of some linear and non-linear parabolic equation. Using idea [1], we will present the explicit unconditional stable scheme which has no restriction on the step size ratio $k / h^{2}$ where $k$ and $h$ are step sizes for space and time respectively. We will also present numerical results to justify the present scheme.
\end{abstract}

\section{Keywords}

Runge-Kutta Methods, Method of Lines, Difference Equation, Non-Linear PDE

\section{Introduction}

A number of difference schemes for solving partial difference equations have been proposed by using the idea of methods of lines [2] [3]. The scheme is required the condition of step size ratio $\frac{k}{h^{2}} \leq c_{0}$ for some constant $c_{0}$, where $k$ and $h$ are step sizes for space and time respectively. We [1] [4]-[6] have proposed some explicit scheme and overcome this problem. The problem considered in this paper is linear and nonlinear parabolic problem

$$
\begin{gathered}
\frac{\partial u(x, t)}{\partial t}=a\{u(x, t)\}^{l} D^{2}(u(x, t)) \quad(l=0,2,4, \cdots, a \text { : positve constant }), \\
(x, t) \in \Omega=\left\{(x, t) ; 0<x<1,0<t \leq t_{F}\right\},
\end{gathered}
$$

with the initial Dirichlet boundary condition

How to cite this paper: Nakashima, M. (2015) Unconditionally Explicit Stable Difference Schemes for Solving Some Linear and Non-Linear Parabolic Differential Equation. Journal of Applied Mathematics and Physics, 3, 1506-1521.

http://dx.doi.org/10.4236/jamp.2015.311176 


$$
u(x, t)= \begin{cases}f(x) & (x, 0) \in \partial \Omega \bigcup \Omega \\ 0, & (0, t),(1, t) \in \partial \Omega \cup \Omega .\end{cases}
$$

where we set

$$
D^{l}=\frac{\partial^{l}}{\partial x^{l}} . \quad(l=1,2,3, \cdots)
$$

We propose the difference approximation to (1.1) where the step size ratio is defined by

$$
c=\frac{k}{h^{2}} . \quad(k \neq 1, \quad c \text { is any positive constant })
$$

The outline of this paper is as follows. In §2, by using idea of methods of lines, we present the explicit difference approximation to (1.1). In $\S 3$ we study the truncation errors of our scheme. In $\S 4$ we study the convergence of the scheme with the condition (1.4) and we will show that our scheme converges to the true solution of (1.1). In $\S 5$ we study stability of the scheme, and we will show that our scheme is stable for any step size $k$ and $h$ with the condition (1.4). In $\S 6$ we show some numerical examples to justify our methods.

\section{Difference Scheme}

In the same way as in [1], we will approximate (1.1) by replacing the derivative for space and time in the difference operator

$$
\begin{gathered}
D^{2}(u(x, t))=\frac{\partial^{2} u(x, t)}{\partial^{2} x} \cong \frac{1}{h^{2}}\left(\delta^{2} u(x, t)\right), \\
\frac{\partial u(x, t)}{\partial x} \cong \frac{1}{h} \Delta u(x, t),
\end{gathered}
$$

where $\delta$ is the central difference operator, $\Delta$ forward difference operator. We denote the approximation to (1.1) at the mesh point $(x, t)=(j h, n k)$

$$
u_{j}^{n} \cong u(j h, n k) .
$$

We set

$$
L(g(x, t))=\{g(x, t)\}^{2 l} .
$$

We define the difference approximation to (1.1) by the following scheme.

If $\left|u_{j}^{n}\right|<1$.

Then we set

$$
u_{j}^{n+1}=u_{j}^{n}+\frac{a c}{1+2 a \hat{c}} \Phi\left(u_{j-1}^{n}, u_{j}^{n}, u_{j+1}^{n}\right) .
$$

If $\left|u_{j}^{n}\right| \geq 1$.

Then we set

$$
u_{j}^{n+1}=u_{j}^{n}+\frac{a c}{1+2 a \hat{c} L\left(u_{j}^{n}\right)} \Phi\left(u_{j-1}^{n}, u_{j}^{n}, u_{j+1}^{n}\right),
$$

where

$$
\Phi\left(u_{j-1}^{n}, u_{j}^{n}, u_{j+1}^{n}\right)=\left\{u_{j}^{n}\right\}^{l}\left\{u_{j-1}^{n}-2 u_{j}^{n}+u_{j+1}^{n}\right\} .
$$

The step size $\hat{c}$ in (2.3), (2.4) is defined by

$$
\hat{c}=\frac{k^{1+\rho}}{h^{2}} . \quad(\rho>0)
$$

If we set 


$$
\tilde{L}\left(u_{j}^{n}\right)= \begin{cases}1 & \left|u_{j}^{n}\right| \leq 1 \\ L\left(u_{j}^{n}\right) & \left|u_{j}^{n}\right|>1 .\end{cases}
$$

Then, from (2.3), (2.4), we have

$$
u_{j}^{n+1}=u_{j}^{n}+\frac{a c}{1+2 a \hat{c} \tilde{L}\left(u_{j}^{n}\right)} \Phi\left(u_{j-1}^{n}, u_{j}^{n}, u_{j+1}^{n}\right) .
$$

\section{Truncation Error}

We define the truncation error $T(j h, n k)$ of (2.8)

$$
\begin{aligned}
T(j h, n k)= & u(j h,(n+1) k)-\left\{u(j h, n k)+\frac{a c}{(1+2 a \hat{c} \tilde{L}(u(j h, n k)))}\right. \\
& \times \Phi(u((j-1) h, n k), u(j h, n k), u((j+1) h, n k))\},
\end{aligned}
$$

where, from the definition of (2.7), we have

$$
\tilde{L}(u(j, n))= \begin{cases}1 & \left|u_{j}^{n}\right| \leq 1 \\ L(u(j, n)) & \left|u_{j}^{n}\right|>1 .\end{cases}
$$

By Taylor series expansions of the solution $u(j h, n k)$ of (1.1), we have

$$
\begin{aligned}
& T(j h, n k)=k u_{t}(j h, n k)+O\left(k^{2}\right) \\
& -\frac{a c}{(1+2 a \hat{c} \tilde{L}(u(j h, n k)))}\{u(j h, n k)\}^{l}\{(u(j-1) h, n k)-2 u(j h, n k)+u((j+1) h, n k)\} \\
& =a k\{u(j h, n k)\}^{l} u_{x x}(j h, n k)-\frac{a k}{(1+2 a \hat{c} \tilde{L}(u(j h, n k)))}\left\{\{u(j h, n k)\}^{l} u_{x x}(j h, n k)\right. \\
& \left.+\frac{k}{12 c}\{u(j h, n k)\}^{l} u_{x x x x}(j h, n k)+O\left(k^{3}\right)\right\} .
\end{aligned}
$$

From (3.3), we have

$$
\begin{aligned}
T(j h, n k)= & k\left\{\frac{2 a^{2} \hat{c} \tilde{L}(u(j h, n k))}{(1+2 a \hat{c} \tilde{L}(u(j h, n k)))}\right\}\{u(j h, n k)\}^{l} u_{x x}(j h, n k) \\
& -\frac{a k^{2}}{12 c(1+2 a \hat{c} \tilde{L}(u(j h, n k)))}\left\{\{u(j h, n k)\}^{l} u_{x x x x}(j h, n k)+O\left(k^{3}\right)\right\} .
\end{aligned}
$$

If we set

$$
\tilde{\rho}=\min \{\rho, 1\},
$$

and

$$
\tilde{c}=\frac{k^{(1+\tilde{\rho})}}{h^{2}} .
$$

Then, from (3.4), we have the following result.

Theorem 1 . The truncation error of the difference approximation (2.8) to (1.1) is given by 


$$
T(j h, n k)=a k^{(1+\tilde{\rho})} w(j h, n k),
$$

where

$$
\begin{aligned}
w(j h, n k)= & \frac{2 a c \tilde{L}(u(j h, n k))}{(1+2 a \hat{c} \tilde{L}(u(j h, n k)))}\{u(j h, n k)\}^{l} u_{x x}(j h, n k) \\
& -k^{(1-\tilde{\rho})} \frac{1}{12 c(1+2 a \hat{c} \tilde{L}(u(j h, n k)))}\{u(j h, n k)\}^{l} u_{x x x x}(j h, n k),
\end{aligned}
$$

where $\tilde{\rho}$ and $\tilde{c}$ are defined by (3.5) and (3.6) respectively.

\section{Convergence}

In this section, we study the convergence of the scheme (2.8). We set the approximation error by

$$
e(j h, n k)=u(j h, n k)-u_{j}^{n} \text {. }
$$

We use the abbreviation's

$$
\begin{gathered}
e_{j}^{n}=e(j h, n k), \\
T(j, n)=T(j h, n k), \\
u(j, n)=u(j h, n k) .
\end{gathered}
$$

From (2.8), (3.7), (4.1), we have

$$
\begin{aligned}
e_{j}^{n+1}= & e_{j}^{n}+g_{1}(j, n) \Phi(u(j-1, n), u(j, n), u(j+1, n)) \\
& -g_{2}(j, n) \Phi\left(u_{j-1}^{n}, u_{j}^{n}, u_{j+1}^{n}\right)+T(j, n+1) \\
= & e_{j}^{n}+\left(g_{1}(j, n)-g_{2}(j, n)\right) \Phi(u(j-1, n), u(j, n), u(j+1, n)) \\
& +g_{2}(j, n)\left\{\Phi(u(j-1, n), u(j, n), u(j+1, n))-\Phi\left(u_{j-1}^{n}, u_{j}^{n}, u_{j+1}^{n}\right)\right\}+T(j, n+1),
\end{aligned}
$$

with

$$
\begin{aligned}
& g_{1}(j, n)=\frac{a c}{1+2 a \tilde{c} \tilde{L}(u(j, n))}, \\
& g_{2}(j, n)=\frac{a c}{1+2 a \tilde{c} \tilde{L}\left(u_{j}^{n}\right)} .
\end{aligned}
$$

From (2.5), we have

$$
\begin{aligned}
\Phi(u(j-1, n), u(j, n), u(j+1, n))=\{u(j, n)\}^{l}\{u(j-1, n)-2 u(j, n)+u(j+1, n)\}, \\
=h^{2}\{u(j, n)\}^{l} u_{x x}(j, n)+O\left(h^{4}\right), \\
\Phi(u(j-1, n), u(j, n), u(j+1, n))-\Phi\left(u_{j-1}^{n}, u_{j}^{n}, u_{j+1}^{n}\right) \\
=u(j, n)^{l}\{u(j-1, n)-2 u(j, n)+u(j+1, n)\}-\left(u_{j}^{n}\right)^{l}\left\{u_{j}^{n-1}-u_{j}^{n}+u_{j+1}^{n}\right\} \\
=\left\{u(j, n)^{l}-\left(u_{j}^{n}\right)^{l}\right\}\{u(j-1, n)-2 u(j, n)+u(j+1, n)\} \\
+\left(u_{j}^{n}\right)^{l}\left\{(u(j-1, n)-2 u(j, n)+u(j+1, n))-\left(u_{j}^{n-1}-u_{j}^{n}+u_{j+1}^{n}\right)\right\} \\
=h^{2}\left\{u(j, n)^{l}-\left(u_{j}^{n}\right)^{l}\right\} u_{x x}(j, n)+h^{2}\left\{u_{j}^{n}\right\}^{l} e_{x x}(j, n)+O\left(h^{4}\right) .
\end{aligned}
$$


We set the initial conditions of (4.2)

$$
\begin{aligned}
& e_{j}^{0}=0, \\
& e_{j}^{1}=T(j, 1) . \quad(0<j<1 / h)
\end{aligned}
$$

Form (4.2), (4.4), (4.5), (4.6), we have

$$
\begin{aligned}
e_{j}^{n+1}= & e_{j}^{n}+\{u(j, n)\}^{l}\left(g_{1}(j, n)-g_{2}(j, n)\right) h^{2} u_{x x}(j, n) \\
& +g_{2}(j, n) h^{2}\left\{\left\{u(j, n)^{l}-\left(u_{j}^{n}\right)^{l}\right\} u_{x x}(j, n)+\left\{u_{j}^{n}\right\}^{l} e_{x x}(j, n)\right\}+T(j, n+1)+O\left(h^{4}\right) \\
= & e_{j}^{n}+h^{2}\left\{g_{1}(j, n) u(j, n)^{l}-g_{2}(j, n)\left(u_{j}^{n}\right)^{l}\right\} u_{x x}(j, n) \\
& +h^{2} g_{2}(j, n)\left\{u_{j}^{n}\right\}^{l} e_{x x}(j, n)+T(j, n+1)+O\left(h^{4}\right) .
\end{aligned}
$$

From (4.7), we have

$$
\begin{aligned}
e_{j}^{n}= & h^{2} \Sigma_{l=1}^{n-1} p_{1}(j, l) u_{x x}(j, l)\left(\Sigma_{s=1}^{l} T(j, s)\right)+h^{2} \Sigma_{l=1}^{n-1} p_{2}(j, l) D^{2}\left(\Sigma_{s=1}^{l} T(j, s)\right)+\Sigma_{l=1}^{n} T(j, l)+O\left(h^{4}\right) \\
= & h^{2} \Sigma_{l=1}^{n-1} k^{\tilde{\rho}} p_{1}(j, l) u_{x x}(j, l) a k\left(\sum_{s=1}^{l} w(j, s)\right)+h^{2} \Sigma_{l=1}^{n-1} k^{\tilde{\rho}} p_{2}(j, l) a k D^{2}\left(\Sigma_{s=1}^{l} w(j, s)\right) \\
& +a k^{(1+\tilde{\rho})} \Sigma_{l=1}^{n} w(j, l)+O\left(h^{4}\right),
\end{aligned}
$$

with

$$
\begin{gathered}
p_{1}(j, n)=\{u(j, n)\}^{l} g_{1}(j, n)-\left\{u_{j}^{n}\right\}^{l} g_{2}(j, n), \\
p_{2}(j, n)=\frac{a c\left\{u_{j}^{n}\right\}^{(l)}}{1+2 a \tilde{c}\left\{u_{j}^{n}\right\}^{(2 l)}} .
\end{gathered}
$$

We study the coefficients of (4.8) to $l \geq 2$.

Firstly we consider the case

$$
\left|u_{i}^{j}\right| \geq 1
$$

We set

$$
\tilde{c}_{1}=(\tilde{c})^{\frac{1}{2 l}} .
$$

Then from (4.3), (4.12), we have

$$
\begin{aligned}
k^{\tilde{\rho}}\{u(j, n)\}^{l} g_{1}(j, n) & =k^{\tilde{\rho}} \frac{a c\{u(j, n)\}^{(l)}}{1+2 a \tilde{c}\{u(j, n)\}^{(2 l)}}=\left\{\tilde{c}_{1}\right\}^{l} \frac{a\left\{\tilde{c}_{1}\right\}^{l}\{u(j, n)\}^{(l)}}{1+2 a \tilde{c}\{u(j, n)\}^{(2 l)}} \\
= & \left\{\tilde{c}_{1}\right\}^{l} \frac{a\left\{\tilde{c}_{1} u(j, n)\right\}^{l}}{1+2 a\left\{\tilde{c}_{1} u(j, n)\right\}^{(2 l)}} . \\
k^{\tilde{\rho}}\left\{u_{j}^{n}\right\}^{l} g_{2}(j, n) & =k^{\tilde{\rho}} \frac{a c\left\{u_{j}^{n}\right\}^{(l)}}{1+2 a \tilde{c}\left\{u_{j}^{n}\right\}^{(2 l)}}=\left\{\tilde{c}_{1}\right\}^{l} \frac{a\left\{\tilde{c}_{1}\right\}^{l}\left\{u_{j}^{n}\right\}^{(l)}}{1+2 a \tilde{c}\left\{u_{j}^{n}\right\}^{(2 l)}} \\
& =\left\{\tilde{c}_{1}\right\}^{l} \frac{a\left\{\tilde{c}_{1} u_{j}^{n}\right\}^{l}}{1+2 a\left\{\tilde{c}_{1} u_{j}^{n}\right\}^{(2 l)}} .
\end{aligned}
$$

We have the equation 


$$
\begin{gathered}
\left|\frac{a\left\{\tilde{c}_{1} u(j, n)\right\}^{(l)}}{\left(1+2 a\left\{\tilde{c}_{1} u(j, n)\right\}^{(2 l)}\right)}\right| \leq 1, \\
\left|\frac{a\left\{\tilde{c}_{1} u_{j}^{n}\right\}^{(l)}}{\left(1+2 a\left\{\tilde{c}_{1} u_{j}^{n}\right\}^{(2 l)}\right)}\right| \leq 1 .
\end{gathered}
$$

From (4.13), (4.14), (4.15), (4.16), we have

$$
\left|k^{\tilde{\rho}} p_{1}(j, n)\right| \leq\left(\left|\{u(j, n)\}^{l} g_{1}(j, n)\right|+\left|\left\{u_{j}^{n}\right\}^{l} g_{2}(j, n)\right|\right) \leq 2\{\tilde{c}\}^{(l)} .
$$

If we assume

$$
u(x, t) \in C^{\infty}(\partial \Omega \bigcup \Omega) .
$$

Then we have

$$
\left|\frac{\partial^{i+j} u(x, t)}{\partial x^{i} t^{j}}\right| \leq K . \quad(i, j=0,1,2,3, \cdots)(K: \text { constat }) \quad(x, t) \in(\partial \Omega \cup \Omega)
$$

From (3.7), we have

$$
\begin{aligned}
|T(j, n)|= & a k^{(1+\tilde{\rho})}|w(j, n)| \\
= & a k^{(1+\tilde{\rho})} \mid \frac{2 a c \tilde{L}(u(j, n))}{\mid(1+2 a \tilde{c} \tilde{L}(u(j, n)))}\{u(j, n)\}^{l} u_{x x}(j, n) \\
& -k^{(1-\tilde{\rho})} \frac{1}{12 c(1+2 a \tilde{c} \tilde{L}(u(j, n)))}\{u(j, n)\}^{l} u_{x x x x}(j, n) \mid \\
= & a k^{(1+\tilde{\rho})} \mid \frac{2 a c\{u(j, n))\}^{2 l}}{\left(1+2 a \tilde{c}\{u(j, n)\}^{(2 l)}\right)}\{u(j, n)\}^{l} u_{x x}(j, n) \\
& -k^{(1-\tilde{\rho})} \frac{1}{12 c\left(1+2 a \tilde{c}\{u(j, n)\}^{(2 l)}\right)}\{u(j, n)\}^{l} u_{x x x x}(j, n) \mid \\
\leq & a k^{(1+\tilde{\rho})} C_{1}, \quad(j h, n k) \in(\partial \Omega \cup \Omega)
\end{aligned}
$$

with

$$
C_{1}=\left\{2 a c K^{(2 l)}+\frac{1}{12 c} k^{(1-\tilde{\rho})}\right\} K^{(l+1)} .
$$

From (4.20), we have

$$
\sum_{l=1}^{n}|T(j, l)|=\left|\sum_{l=1}^{n} a k^{(1+\tilde{\rho})}\right| w(j, l) \mid<a k^{(1+\tilde{\rho})} n C_{1}, \quad(j h, l k) \in(\Omega \bigcup \partial \Omega)
$$

where $C_{1}$ is defined by (4.21).

We have from the condition (1.1)

$$
n k \leq t_{F} .
$$

From (4.17), (4.20), (4.23), we have 


$$
\begin{aligned}
& h^{2} \sum_{l=1}^{n-1}\left|p_{1}(j, l) u_{x x}(j, l)\right|\left(\sum_{s=1}^{l}|T(j, s)|\right) \\
& \leq h^{2} \Sigma_{l=1}^{n-1} a k^{(1+\tilde{\rho})} n C_{1}\left|p_{1}(j, l) u_{x x}(j, l)\right| \leq \frac{k}{c} \sum_{l=1}^{n-1} 2 a C_{1}\left\{t_{F}\right\} K\left\{\tilde{c}_{(1)}\right\}^{(l)} \\
& \leq \frac{2 a}{C}\left\{t_{F}\right\}^{2} C_{1} K\left\{\tilde{c}_{(1)}\right\}^{(l)}, \quad(j h, l k) \in(\Omega \cup \partial \Omega)
\end{aligned}
$$

where $C_{1}$ is defined by (4.21) .

In the same way to (4.16), from (4.10), we have

$$
\begin{aligned}
\left|k^{\tilde{\rho}} p_{2}(j, n)\right| & =\left|k^{\tilde{\rho}} \frac{a c\left\{u_{j}^{n}\right\}^{(l)}}{1+2 a \tilde{c} \tilde{L}\left(u_{j}^{n}\right)}\right|=\left|\left\{\tilde{c}_{1}\right\}^{(l)} \frac{a\left\{\tilde{c}_{1} u_{j}^{n}\right\}^{(l)}}{1+2 a\left\{\tilde{c}_{1} u_{j}^{n}\right\}^{(2 l)}}\right| \\
& \leq\left\{\tilde{c}_{1}\right\}^{(l)}|| \frac{a\left\{\tilde{c}_{1} u_{j}^{n}\right\}^{(l)}}{1+2 a\left\{\tilde{c}_{1} u_{j}^{n}\right\}^{(2 l)}} \mid \leq\left\{\tilde{c}_{1}\right\}^{(l)} .
\end{aligned}
$$

From (3.8), we have

$$
\begin{aligned}
D^{2}[w(j, n)]= & D^{2}\left\{\frac { 2 a c \{ u ( j , n ) \} ^ { ( 2 l ) } } { ( 1 + 2 a \hat { c } \{ u ( j , n ) \} ^ { ( 2 l ) } ) } \left\{\{u(j, n)\}^{(l)} u_{x x}(j, n)\right.\right. \\
& \left.\left.-\frac{k^{(1-\tilde{\rho})}\{u(j, n)\}^{(2 l)}}{12 c\left(1+2 a \tilde{c}\{u(j, n)\}^{(2 l)}\right)}\{u(j, n)\}^{(l)} u_{x x x x}(j, n)\right\}\right\} .
\end{aligned}
$$

After some complicate computation, we have

$$
\begin{aligned}
& D^{2}\left\{\frac{2 a c \tilde{L}_{2}(u(j, n))}{\left(1+2 a \tilde{c}\{u(j, n)\}^{(2 l)}\right)^{(l)}}\{u(j, n)\}^{(l)} u_{x x}(j, n)\right\} \\
& =\frac{q_{4}(j, n)}{\left(1+2 a \tilde{c}\{u(j, n)\}^{(2 l)}\right)^{4}} u_{x x}(j, n)+2 \frac{q_{5}(j, n)}{\left(1+2 a \tilde{c}\{u(j, n)\}^{(2 l)}\right)^{3}} u_{x x x}(j, n) \\
& +\frac{q_{6}(j, n)}{\left(1+2 a \tilde{c}\{u(j, n)\}^{(2 l)}\right)} u_{x x x x}(j, n),
\end{aligned}
$$

with

$$
\begin{aligned}
& q_{4}(j, n)= 2 a c\left\{3 l u_{x x}(j, n)\{u(j, n)\}^{(3 l-1)}+3 l(3 l-1)\left\{u_{x}(j, n)\right\}^{2}\{u(j, n)\}^{(3 l-2)}\right. \\
&+2 a l \tilde{c} u_{x x}(j, n)\{u(j, n)\}^{(5 l-1)}+2 l(5 l-1) a \tilde{c}\left\{u_{x}(j, n)\right\}^{2}\{u(j, n)\}^{(5 l-2)} \\
&+6 a \tilde{c} l u_{x x}(j, n)\{u(j, n)\}^{(5 l-1)}+6 a \tilde{c} l(3 l-1)\left\{u_{x}(j, n)\right\}^{2}\{u(j, n)\}^{(5 l-2)} \\
&+4 a^{2}\{\tilde{c}\}^{2} l u_{x x}(j, n)\{u(j, n)\}^{(7 l-1)}+4 a^{2}\{\tilde{c}\}^{2} l(5 l-1)\left\{u_{x}(j, n)\right\}^{2}\{u(j, n)\}^{(7 l-2)} \\
&\left.-24 l^{2} a \tilde{c}\left\{u_{x}(j, n)\right\}^{2}\{u(j, n)\}^{(5 l-2)}-16 l^{2} a^{2}\{\tilde{c}\}^{2}\left\{u_{x}(j, n)\right\}^{2}\{u(j, n)\}^{(7 l-1)}\right\}, \\
& q_{5}(j, n)=2 a c\left\{3 l u_{x}(j, n)\{u(j, n)\}^{(3 l-1)}+2 a \tilde{c} l\left\{u_{x}(j, n)\right\}^{2}\{u(j, n)\}^{(5 l-1)}\right\},
\end{aligned}
$$




$$
q_{6}(j, n)=2 a c\{u(j, n)\}^{(3 l)}
$$

From (4.27), we have

$$
\left|D^{2}\left\{\frac{2 a c\{u(j, n)\}^{(2 l)}}{\left(1+2 \tilde{c}\{u(j, n)\}^{(2 l)}\right)}\{u(j, n)\}^{(l)} u_{x x}(j, n)\right\}\right| \leq C_{2}, \quad(j h, n k) \in(\partial \Omega \cup \Omega)
$$

with

$$
\begin{aligned}
C_{2}= & 2 a c\left\{(1+6 l+3 l|3 l-1|) K^{(3 l+1)}+\left(10 a \tilde{c} l+2 a l|5 l-1| \tilde{c}+6 a \tilde{c}|3 l-1|+24 a l^{2} \tilde{c}\right) K^{(5 l+1)}\right. \\
& \left.+\left(4 a^{2} l \tilde{c}^{2}+4 a^{2} l \tilde{c}^{2} l|5 l-1|+16 a^{2} l^{2} \tilde{c}^{2}\right) K^{(7 l+1)}\right\} .
\end{aligned}
$$

From (4.26), we have

$$
\begin{aligned}
& D^{2}\left\{\frac{\{u(j, n)\}^{(2 l)}}{\left(1+2 a \hat{c}\left\{u_{j}^{n}\right\}^{(2 l)}\right)^{(2)}}\{u(j, n)\}^{(l)} u_{x x x x}(j, n)\right\} \\
& =\left\{\frac{q_{7}(j, n)}{\left(1+2 a \tilde{c}\{u(j, n)\}^{(2 l)}\right)^{4}}+\frac{q_{8}(j, n)}{\left(1+2 a \tilde{c}\{u(j, n)\}^{(2 l)}\right)^{3}}\right\} u_{x x x x}(j, n) \\
& +2 \frac{q_{9}(j, n)}{\left(1+2 a \tilde{c}\{u(j, n)\}^{(2 l)}\right)^{2}} u_{x x x x x}(j, n)+\frac{q_{10}(j, n)}{\left(1+2 a \tilde{c}\{u(j, n)\}^{(2 l)}\right)^{(x x x x x x}}(j, n),
\end{aligned}
$$

with

$$
\begin{gathered}
q_{7}(j, n)=l u_{x x}\{u(j, n)\}^{(l-1)}+l(l-1)\left\{u_{x}(j, n)\right\}^{2}\{u(j, n)\}^{(l-2)} \\
-2 a l \tilde{c} u_{x x}(j, n)\{u(j, n)\}^{(3 l-1)}-2 a \tilde{c} l(3 l-1)\left\{u_{x}(j, n)\right\}^{2}\{u(j, n)\}^{(3 l-2)}, \\
q_{8}(j, n)=8 a \tilde{c} l^{2}\left\{u_{x}(j, n)\right\}^{2}\{u(j, n)\}^{(3 l-2)}-16 a^{2} l^{2} \tilde{c}^{2}\left\{u_{x}(j, n)\right\}^{2}\{u(j, n)\}^{(5 l-2)}, \\
q_{9}(j, n)=l u_{x}(j, n)\{u(j, n)\}^{(l-1)}-2 a \tilde{c} l u_{x}(j, n)\{u(j, n)\}^{(3 l-1)}, \\
q_{10}(j, n)=2 c\{u(j, n)\}^{(l)} .
\end{gathered}
$$

From (4.30)

$$
\left|D^{2}\left\{\frac{\{u(j, n)\}^{(3 l)}}{\left(1+2 \tilde{c}\{u(j, n)\}^{(2 l)}\right)} u_{x x x x}(j, n)\right\}\right| \leq C_{3}, \quad(j h, n k) \in(\partial \Omega \cup \Omega)
$$

with

$$
C_{3}=\{1+2 l+l|(l-1)|\} K^{(l+1)}+\left\{4 a \tilde{c} l+2 a \tilde{c} l|3 l-1|+8 a \tilde{c} l^{2}\right\} K^{(3 l+1)}+16 a^{2} l^{2} \tilde{c}^{2} K^{(5 l+1)} .
$$

From (4.26), (4.28), (4.31), we have

$$
\left|D^{2}[w(j, n)]\right| \leq\left\{C_{2}+\frac{k^{(1-\tilde{\rho})}}{12 c} C_{3}\right\} . \quad(j h, n k) \in(\partial \Omega \cup \Omega)
$$

From (4.25), we have 


$$
\begin{aligned}
h^{2} \Sigma_{m=1}^{n-1}\left\{p_{2}(j, m) \Sigma_{s=1}^{m} D^{2}[T(j, s)]\right\} & =h^{2} a k^{(1+\tilde{\rho})} \Sigma_{m=1}^{n-1}\left\{\frac{a c\left\{u_{j}^{m}\right\}^{(l)}}{\left(1+2 a \hat{c}\left\{u_{j}^{m}\right\}^{(2 l)}\right)} \sum_{s=1}^{m} D^{2}[w(j, s)]\right\} \\
& =h^{2} \Sigma_{m=1}^{n-1}\left\{k^{\tilde{\rho}} \frac{a c\left\{u_{j}^{m}\right\}^{(l)}}{\left(1+2 a \hat{c}\left\{u_{j}^{m}\right\}^{(2 l)}\right)} a k \Sigma_{s=1}^{m} D^{2}[w(j, s)]\right\} .
\end{aligned}
$$

From (4.25), (4.33), (4.34), we have

$$
\begin{aligned}
& h^{2} \sum_{m=1}^{n-1}\left|\left\{p_{2}(j, m) \Sigma_{s=1}^{m} D^{2}[T(j, s)]\right\}\right| \\
& \leq \frac{k}{c} \sum_{m=1}^{n-1}\left\{\tilde{c}_{1}\right\}^{l} \mid a k \Sigma_{s=1}^{m} D^{2}[w(j, s)] \leq \frac{k}{c}\left(n\left\{\tilde{c}_{1}\right\}^{l}\right) a k \sum_{m=1}^{n-1}\left(C_{2}+\frac{k^{(1-\tilde{\rho})}}{12 c} C_{3}\right) \\
& \leq \frac{a}{c}\left\{\tilde{c}_{1}\right\}^{l}\left\{t_{F}\right\}^{2}\left(C_{2}+\frac{k^{(1-\tilde{\rho})}}{12 c} C_{3}\right), \quad(j h, l k) \in(\Omega \cup \partial \Omega)
\end{aligned}
$$

where $C_{2}$ and $C_{3}$ are defined by (4.29) and (4.32) respectively.

From (4.20), we have

$$
\begin{aligned}
\sum_{l=1}^{n}|T(j, l)| & =a k^{(1+\tilde{\rho})}\left|\sum_{l=1}^{n} w(j, l)\right| \leq a k \sum_{l=1}^{n} k^{\tilde{\rho}}|w(j, l)| \\
& \leq a k^{\tilde{\rho}}\left\{t_{F}\right\} C_{1}+0\left(k^{2}\right), \quad(j h, l k) \in(\partial \Omega \cup \Omega)
\end{aligned}
$$

where $C_{1}$ is defined by (4.21).

From (4.8), (4.20) (4.24), (4.35), (4.36), we have

$$
\begin{aligned}
\left|e_{j}^{n}\right| \leq & h^{2} \Sigma_{l=1}^{n-1}\left|p_{1}(j, l) u_{x x}(j, l)\right|\left(\Sigma_{s=1}^{l}|T(j, s)|\right) \\
& +h^{2} \Sigma_{l=1}^{n-1}\left|p_{2}(j, l)\right|\left\{D^{2}\left(\Sigma_{s=1}^{l}|T(j, s)|\right)\right\}+\Sigma_{l=1}^{n}|T(j, l)|+O\left(h^{4}\right) \\
\leq & \frac{2 a}{c}\left\{t_{F}\right\}^{(2)} C_{1} K\left\{\tilde{c}_{1}\right\}^{(l)}+\frac{a}{c}\left\{t_{F}\right\}^{2}\left(C_{2}+\frac{k^{(1-\tilde{\rho})}}{12 c} C_{3}\right)\left\{\tilde{c}_{1}\right\}^{l} \\
& +a k^{\tilde{\rho}} t_{F} C_{1}+0\left(k^{2}\right), \quad(j h, l k) \in(\Omega \cup \partial \Omega)
\end{aligned}
$$

where $C_{1}, C_{2}$ and $C_{3}$ are defined by (4.21), (4.29) and (4.32) respectively.

We set the maximum norm of the function $e_{j}^{n}$

$$
\left\|E^{n}\right\|=\max _{1 \leq j \leq 1 / k}\left|e_{j}^{n}\right| .
$$

Then, from (4.37), we have

$$
\left\|E^{n}\right\|<\frac{2 a}{c}\left\{t_{F}\right\}^{(2)} C_{1} K\left\{\tilde{c}_{1}\right\}^{(l)}+\frac{a}{c}\left\{\tilde{c}_{1}\right\}^{l}\left\{t_{F}\right\}^{2}\left(C_{2}+\frac{k^{(1-\tilde{\rho})}}{12 c} C_{3}\right)+a k^{\tilde{\rho}} t_{F} C_{1}+0\left(k^{2}\right) .
$$

From (4.39), we have

$$
\lim _{k \rightarrow 0}\left\|E^{n}\right\|=0
$$

Finally we assume

$$
\left|u_{j}^{n}\right| \leq 1
$$

Then, from (4.3), we have

$$
g_{1}(j, n)=g_{2}(j, n)
$$

From (4.9), (4.42), we have 


$$
\begin{aligned}
\left|p_{1}(j, n)\right| & =\left|\{u(j, n)\}^{l} g_{1}(j, n)-\left\{u_{j}^{n}\right\}^{l} g_{2}(j, n)\right| \\
& =\left|\frac{a c}{(1+2 a \tilde{c})}\left\{\{u(j, n)\}^{l}-\left\{u_{j}^{n}\right\}^{l}\right\}\right| \leq a c\{K\}^{(l)} . \quad(j h, l k) \in(\partial \Omega \cup \Omega)
\end{aligned}
$$

In the same way to (4.14), we have

$$
\left|p_{2}(j, n)\right|=\left|\frac{a c\left\{u_{j}^{n}\right\}^{(l)}}{1+2 a \tilde{c}}\right| \leq a c .
$$

From (3.8), we have after some computation,

$$
\left|D^{2}\left\{w_{2}(j, n) u_{x x}(j, n)\right\}\right| \leq C_{4}, \quad(j h, n k) \in(\partial \Omega \cup \Omega)
$$

with

$$
C_{4}=\frac{2 a c}{(1+2 a \tilde{c})}\{l|l-1|+3 l+1\}\left\{1+\frac{1}{24 a c^{2}} k^{(1-\tilde{\rho})}\right\} K^{(l+1)} .
$$

From (4.8), (4.20), (4.43), (4.44), (4.45), we have

$$
\begin{aligned}
\left|e_{j}^{n}\right| \leq & h^{2} \Sigma_{l=1}^{n-1}\left|p_{1}(j, l) u_{x x}(j, l) a k^{(1+\tilde{\rho})}\left(\Sigma_{s=1}^{l} w(j, s)\right)\right| \\
& +h^{2} \Sigma_{l=1}^{n-1}\left|p_{2}(j, l) a k^{(1+\tilde{\rho})} D^{2}\left(\Sigma_{s=1}^{l} w(j, s)\right)\right|+a k^{(1+\tilde{\rho})} \sum_{l=1}^{n}|w(j, l)|+O\left(h^{4}\right) \\
\leq & \left(\frac{k}{c}\right) \Sigma_{l=1}^{n-1} a c k^{\tilde{\rho}}\{K\}^{(l+1)} a\left\{t_{F}\right\} C_{1}+\left(\frac{k}{C}\right) \Sigma_{l=1}^{n-1} a c a k^{(1+\tilde{\rho})} D^{2}\left(\Sigma_{s=1}^{n} w(j, s)\right)+a k^{(1+\tilde{\rho})} n C_{1}+O\left(h^{4}\right) . \\
< & a^{2}\left\{t_{F}\right\}^{(2)}\{K\}^{(l+1)} C_{1} k^{\tilde{\rho}}+a^{2}\left\{t_{F}\right\}^{(2)} C_{4} k^{\tilde{\rho}}+a k^{\tilde{\rho}}\left\{t_{F}\right\} C_{1}+O\left(h^{4}\right) . \quad(j h, l k) \in(\partial \Omega \cup \Omega)
\end{aligned}
$$

where $C_{1}$ and $C_{4}$ are defined by (4.21) and (4.46) respectively.

Then, in the same way to (4.40), from (4.47), we have

$$
\lim _{k \rightarrow 0}\left\|E^{n}\right\|=0 .
$$

We study $l=0$. In the almost same way to (4.47), we have

$$
\left|e_{j}^{n}\right| \leq a^{2}\left\{t_{F}\right\}^{(2)} C_{4} k^{\tilde{p}}+a k^{\tilde{p}}\left\{t_{F}\right\} C_{1}+O\left(h^{4}\right) . \quad(j h, l k) \in(\partial \Omega \bigcup \Omega)
$$

where $C_{1}$ and $C_{4}$ are defined by (4.21) and (4.46) with $l=0$ respectively.

From (4.49), we have

$$
\lim _{k \rightarrow 0}\left\|E^{n}\right\|=0 .
$$

From (4.40), (4.48), (4.50), we have

Theorem 2. Suppose that for $h_{i}$ and $k_{i}$, there exists positive numbers $j\left(h_{i}\right)$ and $n\left(k_{i}\right)$ such that

$$
j\left(h_{i}\right) h_{i} \rightarrow x \in[0,1](i \rightarrow \infty) \quad n\left(k_{i}\right) k_{i} \rightarrow t \in\left[0, t_{F}\right] .
$$

If the solution $u(x, t)$ of (1.1) satisfies conditions (4.18). Then, the approximation $u_{j}^{n}$ generated by the scheme (2.8) converges to the solution $u(x, t)$ of the differential Equation (1.1).

\section{Stability}

In this section, we study the stability of the numerical process (2.8) and define as follows.

Definition: The numerical processes $\left\{Y^{n} \in R_{n}\right\}$ is stable if there exists a positive constant $K_{2}$ such that

$$
\left\|Y^{n}\right\| \leq K_{2} \text {, }
$$

where $\|\cdot\|$ denotes some norm and the constant $K_{2}$ is depends on initial value. 
We prove that the scheme (2.8) are stable in mean of the von Neumann.

We set

$$
x=j h .
$$

Then, from (4.7), we have

$$
\begin{aligned}
e(x, n)= & h^{2} \sum_{l=1}^{n-1} k^{\tilde{\rho}} p_{1}(x, l) u_{x x}(x, l) a k\left(\sum_{s=1}^{l} w(x, s)\right) \\
& +\sum_{l=1}^{n-1} k^{\tilde{\rho}} p_{2}(x, l) a k D^{2}\left(\sum_{s=1}^{l} w(x, s)\right) \\
& +a k^{(1+\tilde{\rho})} \sum_{s=1}^{n} w(x, s)+O\left(h^{4}\right) .
\end{aligned}
$$

From (5.1), we have

$$
\begin{aligned}
& D^{(2)}\{e(x, n)\}=h^{2} \Sigma_{l=1}^{n-1} k^{\tilde{\rho}}\left\{\left(D^{(2)}\left(p_{1}(x, l)\right)\right) u_{x x}(j, l) k\left(\sum_{s=1}^{l} w(x, s)\right)\right. \\
& +p_{1}(x, l)\left(D^{(2)} u_{x x}(x, l)\right) k\left(\Sigma_{s=1}^{l} w(x, s)\right)+p_{1}(x, l) u_{x x}(x, l) k\left(\sum_{s=1}^{l} D^{(2)}(w(x, s))\right) \\
& +2\left(D\left(p_{1}(x, l)\right)\left(D\left(u_{x x}(x, l)\right)\right) k\left(\Sigma_{s=1}^{l} w(x, s)\right)+\left(D p_{1}(x, l)\right) u_{x x}(x, l) k\left(\Sigma_{s=1}^{l} D(w(x, s))\right)\right. \\
& \left.\left.+p_{1}(x, l)\left(D u_{x x}(x, l)\right) k\left(\Sigma_{s=1}^{l} D(w(x, s))\right)\right)\right\}+\Sigma_{l=1}^{n-1} k^{\tilde{\rho}}\left\{( D ^ { ( 2 ) } p _ { 2 } ( x , l ) ) k \left(\Sigma_{s=1}^{l}\left(D^{(2)}(w(x, s))\right)\right.\right. \\
& \left.+2\left(D p_{2}(x, l)\right) k\left(\Sigma_{s=1}^{l} D^{(3)}(w(x, s))\right)+p_{2}(x, l) k\left(\Sigma_{s=1}^{l} D^{(4)}(w(x, s))\right)\right\} \\
& +h^{(1+\tilde{\rho})} \Sigma_{l=1}^{n}\left(D^{(2)} w(x, s)\right)+O\left(h^{4}\right),
\end{aligned}
$$

where $p_{1}(x, n), p_{2}(x, n)$ and $w(x, n)$ are defined by (4.9), (4.10) and (3.8) respectively.

If we assume (4.18) on the solution $u(t, x)$ of (1.1), Then,in the same way to (4.31), (4.33), (4.45), we have

$$
\begin{aligned}
& \left|h^{2} \Sigma_{l=1}^{n-1} k^{\tilde{\rho}}\left(D^{(2)} p_{1}(x, l)\right) u_{x x}(x, l) k\left(\Sigma_{s=1}^{l} w(x, s)\right)\right| \leq C_{5}, \\
& \left|h^{2} \Sigma_{l=1}^{n-1} p_{1}(x, l)\left(D^{(2)} u_{x x}(x, l)\right) k\left(\Sigma_{s=1}^{l} w(x, s)\right)\right| \leq C_{5}, \\
& \left|h^{2} \Sigma_{l=1}^{n-1} p_{1}(x, l) u_{x x}(x, l) k\left(\Sigma_{s=1}^{l}\left(D^{(2)}(w(x, s))\right)\right)\right| \leq C_{5}, \\
& \left|h^{2} \Sigma_{l=1}^{n-1}\left(D\left(p_{1}(x, l)\right)\right)\left(D\left(u_{x x}(x, l)\right)\right) k\left(\Sigma_{s=1}^{l} w(x, s)\right)\right| \leq C_{5}, \\
& \left|h^{2} \Sigma_{l=1}^{n-1}\left(D p_{1}(x, l)\right) u_{x x}(x, l) k\left(\sum_{s=1}^{l} D(w(x, s))\right)\right| \leq C_{5}, \\
& \left|h^{2} \Sigma_{l=1}^{n-1} p_{1}(x, l)\left(D u_{x x}(x, l)\right) k\left(\sum_{s=1}^{l} D(w(x, s))\right)\right| \leq C_{5}, \\
& \mid \Sigma_{l=1}^{n-1} k^{\tilde{\rho}}\left(D^{(2)} p_{2}(x, l)\right) k\left(\Sigma_{s=1}^{l}\left(D^{(2)}(w(x, s))\right) \mid \leq C_{5}\right. \text {, } \\
& \left|\Sigma_{l=1}^{n-1} k^{\tilde{\rho}}\left(D p_{2}(x, l)\right) k\left(\Sigma_{s=1}^{l}\left(D^{(3)}(w(x, s))\right)\right)\right| \leq C_{5} \text {, } \\
& \left|\sum_{l=1}^{n-1} k^{\tilde{\rho}} p_{2}(x, l) k\left(\Sigma_{s=1}^{l}\left(D^{(4)}(w(x, s))\right)\right)\right| \leq C_{5}, \quad(x, l k) \in(\partial \Omega \cup \Omega)
\end{aligned}
$$

for some constant $C_{5}$.

From (5.2), (5.3), we have the following result.

Lemma 1. If we assume the solution $u(x, t)$ of (1.1) satisfies (4.18), Then there exists the constant $C_{5}$ such that

$$
\begin{gathered}
\left|\frac{\partial^{2}}{\partial x^{2}} e(x, j)\right| \leq C_{6}, \quad(x, j k) \in(\partial \Omega \cup \Omega) \\
\text { with } C_{6}=6 C_{5},
\end{gathered}
$$

where $C_{5}$ is defined by (5.3). From (2.8), we have 


$$
\left|u_{j}^{n+1}\right| \leq\left|u_{j}^{n}+\frac{a c}{1+2 a c \tilde{L}\left(u_{j}^{n}\right)} \Phi\left(u_{j-1}^{n}, u_{j}^{n}, u_{j+1}^{n}\right)\right|+\left|\frac{a c}{1+2 a \hat{c} \tilde{L}(u(j h, n k))} \Phi\left(u_{j-1}^{n}, u_{j}^{n}, u_{j+1}^{n}\right)-\frac{a c}{1+2 a c \tilde{L}\left(u_{j}^{n}\right)} \Phi\left(u_{j-1}^{n}, u_{j}^{n}, u_{j+1}^{n}\right)\right| .
$$

We set the maximum norm of the function $u_{j}^{n}$

$$
\left\|U^{n}\right\|=\max _{1 \leq j \leq 1 / k}\left|u_{j}^{n}\right| .
$$

We have the inequality

$$
\begin{aligned}
& \left|u_{j}^{n}+\frac{a c}{1+2 a c \tilde{L}\left(u_{j}^{n}\right)} \Phi\left(u_{j-1}^{n}, u_{j}^{n}, u_{j+1}^{n}\right)\right| \\
& =\left|u_{j}^{n}+\frac{a c}{1+2 a c \tilde{L}\left(u_{j}^{n}\right)}\left\{u_{j}^{n}\right\}^{l}\left\{u_{j-1}^{n}-2 u_{j}^{n}+u_{j+1}^{n}\right\}\right| \\
& \leq\left|\left(1-2 \frac{a c\left\{u_{j}^{n}\right\}^{l}}{1+2 a c \tilde{L}\left(u_{j}^{n}\right)}\right)\right|\left|u_{j}^{n}\right|+\left|\frac{a c\left\{u_{j}^{n}\right\}^{l}}{1+2 a c \tilde{L}\left(u_{j}^{n}\right)^{n}} u_{j-1}^{n}+\frac{a c\left\{u_{j}^{n}\right\}^{l}}{1+2 a c \tilde{L}\left(u_{j}^{n}\right)} u_{j+1}^{n}\right| \\
& \leq\left\|U^{n}\right\|+2 \frac{a c\left(\left|\left\{u_{j}^{n}\right\}^{l}\right|-\left\{u_{j}^{n}\right\}^{l}\right)}{1+2 a c \tilde{L}\left(u_{j}^{n}\right)} \mid U^{n} \| .
\end{aligned}
$$

From (1.1), we have

$$
\left\{u_{j}^{n}\right\}^{l} \geq 0
$$

From (5.8), we have

$$
\left|u_{j}^{n}+\frac{a c}{1+2 a c \tilde{L}\left(u_{j}^{n}\right)} \Phi\left(u_{j-1}^{n}, u_{j}^{n}, u_{j+1}^{n}\right)\right| \leq\left\|U^{n}\right\| .
$$

From (2.8), we have

$$
\begin{aligned}
& \frac{a c}{\left(1+2 a \tilde{c} \tilde{L}\left(u_{j}^{n}\right)\right)} \Phi\left(u_{j-1}^{n}, u_{j}^{n}, u_{j+1}^{n}\right)-\frac{a c}{\left(1+2 a c \tilde{L}\left(u_{j}^{n}\right)\right)} \Phi\left(u_{j-1}^{n}, u_{j}^{n}, u_{j+1}^{n}\right) \\
& =a c\left\{\frac{1}{1+2 a \tilde{c} \tilde{L}\left(u_{j}^{n}\right)}-\frac{1}{1+2 a c \tilde{L}\left(u_{j}^{n}\right)}\right\} \Phi\left(u_{j-1}^{n}, u_{j}^{n}, u_{j+1}^{n}\right) . \\
& =\left(a^{2}\right) \frac{2 c(c-\tilde{c}) \tilde{L}\left(u_{j}^{n}\right)}{\left(1+2 a \tilde{L} \tilde{L}\left(u_{j}^{n}\right)\right)\left(1+2 a c \tilde{L}\left(u_{j}^{n}\right)\right)}\left\{u_{j}^{n}\right\}^{l}\left\{u_{j-1}^{n}-2 u_{j}^{n}+u_{j+1}^{n}\right\},
\end{aligned}
$$

and

$$
\begin{aligned}
\left\{u_{j-1}^{n}-2 u_{j}^{n}+u_{j+1}^{n}\right\} & =\left(u(j-1, n)-e_{j-1}^{n}\right)-2\left(u(j, n)-e_{j}^{n}\right)+\left(u(j+1, n)-e_{j+1}^{n}\right) \\
& =h^{2}\left(u_{x x}(j, n)-e_{x x}(j, n)\right)+O\left(h^{4}\right) .
\end{aligned}
$$

From (5.10), (5.11), we have

$$
\begin{aligned}
& \left|\frac{a c}{\left(1+2 a \tilde{c} \tilde{L}\left(u_{j}^{n}\right)\right)} \Phi\left(u_{j-1}^{n}, u_{j}^{n}, u_{j+1}^{n}\right)-\frac{a c}{\left(1+2 a c \tilde{L}\left(u_{j}^{n}\right)\right)} \Phi\left(u_{j-1}^{n}, u_{j}^{n}, u_{j+1}^{n}\right)\right| \\
& =\left(a^{2}\right)\left|k \frac{2(c-\tilde{c}) \tilde{L}\left(u_{j}^{n}\right)}{\left(1+2 a \tilde{c} \tilde{L}\left(u_{j}^{n}\right)\right)\left(1+2 a c \tilde{L}\left(u_{j}^{n}\right)\right)}\left\{u_{j}^{n}\right\}^{l}\left(u_{x x}(j, n)-e_{x x}(j, n)\right)+O\left(h^{2}\right)\right| .
\end{aligned}
$$


Firstly we consider

$$
l=0 .
$$

Then from (5.9) and (5.12), we have

$$
\left|u_{j}^{n+1}\right| \leq\left\|U^{n}\right\|+C_{7} k+O\left(k^{2}\right)
$$

with

$$
C_{7}=\left(2 a^{2}\right)(c-\tilde{c})\left(K+C_{6}\right)
$$

where $K, C_{6}$ are defined by (4.19) and (5.5) respectively.

From (5.14), we have

$$
\left\|U^{n}\right\| \leq C_{7} t_{F}+O(k)
$$

Lastly, we consider

$$
l \geq 2
$$

From (5.12), we have

$$
\begin{aligned}
& \left|\frac{a c}{\left(1+2 a \tilde{c} \tilde{L}\left(u_{j}^{n}\right)\right)} \Phi\left(u_{j}^{n}, u_{j}^{n}, u_{j+1}^{n}\right)-\frac{a c}{\left(1+2 a c \tilde{L}\left(u_{j}^{n}\right)\right)} \Phi\left(u_{j}^{n}, u_{j}^{n}, u_{j+1}^{n}\right)\right| \\
& =\left|a(c-\tilde{c}) \frac{1}{\left(1+2 a \tilde{c} \tilde{L}\left(u_{j}^{n}\right)\right)} \frac{2 a c \tilde{L}\left(u_{j}^{n}\right)}{\left(1+2 a c \tilde{L}\left(u_{j}^{n}\right)\right)}\left\{u_{j}^{n}\right\}^{l} h^{(2)}\left(u_{x x}(j, n)-e_{x x}(j, n)\right)+O\left(h^{2}\right)\right| \\
& \left|a \frac{k}{c}(c-\tilde{c})\left\{\tilde{c}_{1}\right\}^{-(l)}\left(\frac{\left\{\tilde{c}_{1}\right\}^{l}\left\{u_{j}^{n}\right\}^{(l)}}{\left(1+2 a \tilde{c} \tilde{L}\left(u_{j}^{n}\right)\right)}\right)\left(\frac{2 a c \tilde{L}\left(u_{j}^{n}\right)}{\left(1+2 a c \tilde{L}\left(u_{j}^{n}\right)\right)}\right)\left(u_{x x}(j, n)-e_{x x}(j, n)\right)+O\left(h^{2}\right)\right| .
\end{aligned}
$$

Firstly, we consider the case $\left|u_{j}^{n}\right| \geq 1$.

Then from (5.16), we have

$$
\begin{aligned}
& \left|\frac{a c}{\left(1+2 a \tilde{c} \tilde{L}\left(u_{j}^{n}\right)\right)} \Phi\left(u_{j}^{n}, u_{j}^{n}, u_{j+1}^{n}\right)-\frac{a c}{\left(1+2 a c \tilde{L}\left(u_{j}^{n}\right)\right)} \Phi\left(u_{j}^{n}, u_{j}^{n}, u_{j+1}^{n}\right)\right| \\
& =\left|a \frac{k}{c}(c-\tilde{c})\left\{\tilde{c}_{1}\right\}^{-(l)}\left(\frac{\left\{\tilde{c}_{1} u_{j}^{n}\right\}^{(l)}}{\left(1+2 a\left\{\tilde{c}_{(1)} u_{j}^{n}\right\}^{(2 l)}\right)}\right)\left(\frac{2 a c\left\{u_{j}^{n}\right\}^{(2 l)}}{\left(1+2 a c\left\{u_{j}^{n}\right\}^{(2 l)}\right)}\right)\left(u_{x x}(j, n)-e_{x x}(j, n)+O\left(h^{2}\right)\right)\right|, \\
& \leq\left|\frac{k}{2 c}(c-\tilde{c})\left\{c_{1}\right\}^{-(l)}\left(\frac{2 a\left\{\tilde{c}_{1} u_{j}^{n}\right\}^{(l)}}{\left(1+2 a\left\{\tilde{c}_{(1)} u_{j}^{n}\right\}^{(2 l)}\right)}\right)\left(\frac{2 a c\left\{u_{j}^{n}\right\}^{(2 l)}}{\left(1+2 a c\left\{u_{j}^{n}\right\}^{(2 l)}\right)}\right)\left(u_{x x}(j, n)-e_{x x}(j, n)+O\left(h^{2}\right)\right)\right| u_{j}^{n} \mid .
\end{aligned}
$$

We have

$$
\left|\frac{2 a\left\{\tilde{c}_{1} u_{j}^{n}\right\}^{(l)}}{\left(1+2 a\left\{\tilde{c}_{(1)} u_{j}^{n}\right\}^{(2 l)}\right.}\right| \leq 1, \quad\left|\frac{2 a c\left\{u_{j}^{n}\right\}^{(2 l)}}{\left(1+2 a c\left\{u_{j}^{n}\right\}^{(2 l)}\right)}\right| \leq 1 .
$$

From (5.10). (5.17), (5.18), we have

$$
\left|\frac{a c}{\left(1+2 a \tilde{c} \tilde{L}\left(u_{j}^{n}\right)\right)} \Phi\left(u_{j-1}^{n}, u_{j}^{n}, u_{j+1}^{n}\right)-\frac{a c}{\left(1+2 a c \tilde{L}\left(u_{j}^{n}\right)\right)} \Phi\left(u_{j-1}^{n}, u_{j}^{n}, u_{j+1}^{n}\right)\right| \leq\left(\frac{1}{2} k C_{8}+O\left(k^{2}\right)\right)\left|u_{j}^{n}\right| \text {, }
$$


with

$$
\begin{aligned}
C_{8} & =\left|\frac{1}{c}(c-\tilde{c})\left\{\tilde{c}_{1}\right\}^{-(l)}\right|\left(K+C_{6}\right) \\
& =\left|\frac{\left(1-k^{\rho}\right)}{\sqrt{c} \sqrt{k}^{\rho}}\right|\left(K+C_{6}\right),
\end{aligned}
$$

where $K$ and $C_{6}$ are defined by (4.19) and (5.5) respectively.

If $0<k<1$, Then we set

$$
\rho=\log _{k}\left(1-(\sqrt{c}) S_{1}\right) . \quad\left(0<S_{1}<\frac{(1-k)}{(\sqrt{c})}\right)
$$

From (5.21), we have

$$
\frac{\left(1-k^{\rho}\right)}{\sqrt{c} \sqrt{k^{\rho}}}=\frac{S_{1}}{\sqrt{\left(1-(\sqrt{c}) S_{1}\right)}} .
$$

If $k>1$, Then we set

$$
\rho=\log _{k}\left(1+(\sqrt{c}) S_{2}\right) \cdot\left(0<S_{2}<\frac{(k-1)}{(\sqrt{c})}\right)
$$

From (5.23), we have

$$
\frac{\left(k^{\rho}-1\right)}{\sqrt{c} \sqrt{k^{\rho}}}=\frac{S_{2}}{\sqrt{\left(1+(\sqrt{c}) S_{2}\right)}} .
$$

From (5.22), (5.24), we set

$$
C_{9}= \begin{cases}\frac{S_{1}}{\sqrt{\left(1-(\sqrt{c}) S_{1}\right)}}\left(K+C_{6}\right) & (0<k<1) \\ \frac{S_{2}}{\sqrt{\left(1+(\sqrt{c}) S_{2}\right)}}\left(K+C_{6}\right), & (k>1) .\end{cases}
$$

where $S_{1}$ and $S_{2}$ are satisfy (5.21) and (5.23) respectively.

From (5.6), (5.19) and (5.25), we have

$$
\left|u_{n+1}^{j}\right| \leq\left(1+\frac{k}{2} C_{9}+O\left(k^{2}\right)\right)\left\|U^{n}\right\|,
$$

and we have the following result

$$
\left\|U^{n+1}\right\| \leq\left(1+\frac{k}{2} C_{9}+O\left(k^{2}\right)\right)\left\|U^{n}\right\| .
$$

From (5.26), we have

$$
\left\|U^{n}\right\| \leq \mathrm{e}^{\left(\frac{1}{2} C_{9} t_{F}\right)}\left\|U^{0}\right\|,
$$

where $C_{9}$ is defined by (5.25).

Secondly, in the case $\left|u_{j}^{n}\right| \leq 1$, from (5.12), we have 


$$
\begin{aligned}
& \left|\frac{a c}{\left(1+2 a \tilde{L} \tilde{L}\left(u_{j}^{n}\right)\right)} \Phi\left(u_{j-1}^{n}, u_{j}^{n}, u_{j+1}^{n}\right)-\frac{a c}{\left(1+2 a c \tilde{L}\left(u_{j}^{n}\right)\right)} \Phi\left(u_{j-1}^{n}, u_{j}^{n}, u_{j+1}^{n}\right)\right| \\
& =\left(a^{2}\right) c\left|\frac{2(c-\tilde{c})}{(1+2 a \tilde{c})(1+2 a c)} h^{2}\left\{u_{j}^{n}\right\}^{l}\left(u_{x x}(j, n)-e_{x x}(j, n)+O\left(h^{2}\right)\right)\right| .
\end{aligned}
$$

From (5.28), we have

$$
\left|\frac{a c}{\left(1+2 a \tilde{c} \tilde{L}\left(u_{j}^{n}\right)\right)} \Phi\left(u_{j-1}^{n}, u_{j}^{n}, u_{j+1}^{n}\right)-\frac{a c}{\left(1+2 a c \tilde{L}\left(u_{j}^{n}\right)\right)} \Phi\left(u_{j-1}^{n}, u_{j}^{n}, u_{j+1}^{n}\right)\right| \leq k C_{10}\left|u_{j}^{n}\right|
$$

with

$$
C_{10}=2 a^{2}|(c-\tilde{c})|\left(K+C_{6}\right),
$$

where $K$ and $C_{6}$ are defined by (4.19)and (5.5) respectively.

In the same way to (5.26), we have

$$
\left\|U^{n}\right\| \leq \mathrm{e}^{\left(C_{10} t_{F}\right)}\left\|U^{0}\right\|
$$

where $C_{10}$ is defined by (5.30).

From (5.15), (5.27), (5.31), we have

Theorem 3.

If the solution $u(t, x)$ of (1.1) is analytic on the region $\partial \Omega \cup \Omega$ then the difference approximation (2.8) to (1.1) are stable.

\section{Numerical Example}

Lastly, we study the numerical test in the following non-linear Equation .

$$
\frac{\partial u(x, t)}{\partial t}=\{u(x, t)\}^{2} \frac{\partial^{2} u(x, t)}{\partial^{2} x}
$$

and the initial and boundary problem given by,

$$
\begin{aligned}
& u(x, 0)= \begin{cases}4 x^{2} & \left(0 \leq x \leq \frac{1}{2}\right) \\
4(1-x)^{2}, & \left(\frac{1}{2} \leq x \leq 1\right)\end{cases} \\
& u_{t}(0, t)=u(1, t)=0 .
\end{aligned}
$$

Applying the difference Equation (2.8) to (6.1) with (6.2), we have the the numerical results in Table 1 and Figure 1, Figure 2.

Table 1. $(\mathrm{x}=0 / 100,2 / 100,20 / 100,50 / 100,70 / 100,98 / 100),(\mathrm{t}=0,2 / 100$,
10/100, 20/100, 50/100).
$\begin{array}{ccccccc} & x & 2 h & 20 h & 50 h & 70 h & 98 h \\ t & 0 & 0.4 E-3 & 0.336 E+0 & 0.1 E+1 & 0.384 E+0 & 0.4 E-3 \\ t & 2 & 0.4 E-3 & 0.336 E+00 & 0.997 E+00 & 0.384 E+00 & 0.4 E-3 \\ t & 10 & 0.4 E-3 & 0.336 E+00 & 0.986 E+00 & 0.384 E+00 & 0.4 E-3 \\ t & 20 & 0.4 E-3 & 0.336 E+00 & 0.981 E+00 & 0.384 E+00 & 0.4 E-3 \\ t & 50 & 0.4 E-3 & 0.336 E+00 & 0.964 E+00 & 0.384 E+00 & 0.4 E-3\end{array}$




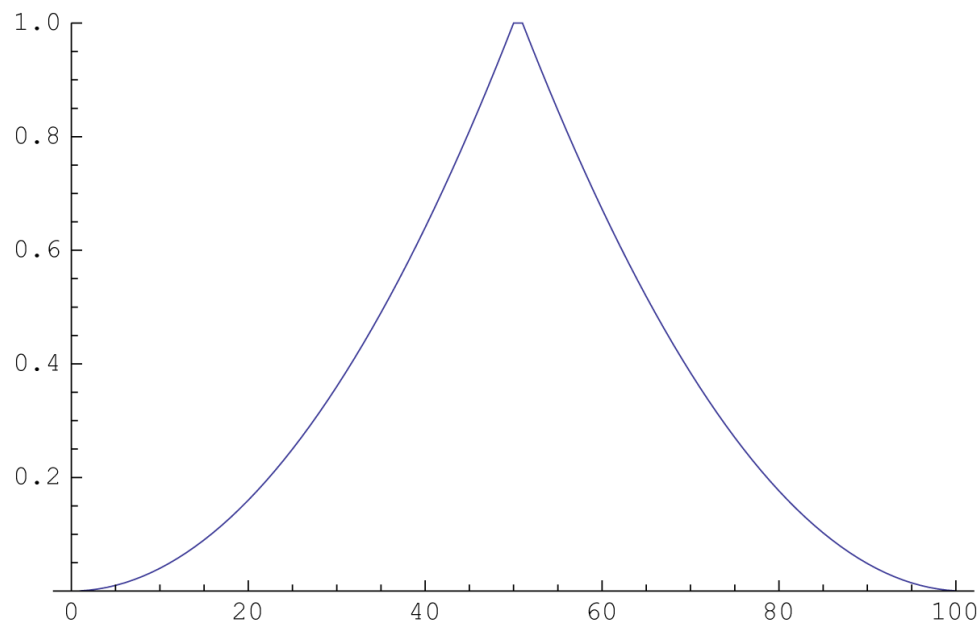

Figure 1. Initial data $(0 \leq \mathrm{x} \leq 1, t=0)$.

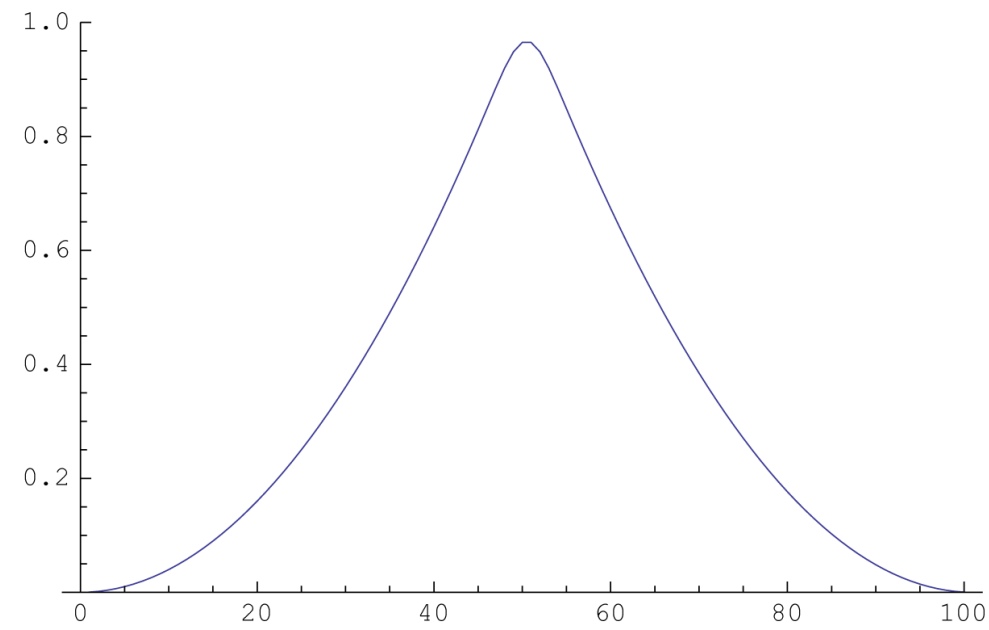

Figure 2. The numerical solution for $0 \leq \mathrm{x} \leq 1, t=50$.

As we see in Figure 1, Figure 2, the initial data diffuses slowly. Here the interval $[0,1]$ is divided into $M=100$ with $h=\frac{1}{100}, k=\frac{1}{100}, \rho=\frac{1}{200}$.

\section{References}

[1] Nakashima, M. (2013) A Study on Unconditionally Stable Explicit Difference Schemes for the Variable Coefficients Parabolic Differential Equation. IJPAM, 87, 587-602. http://dx.doi.org/10.12732/ijpam.v87i4.8

[2] Du Fort, E.G. and Frankel, E.G. (1953) Stability Conditions in the Numerical Treatment on Parabolic Differential Equations. Mathematical Tables and Other Aids to Computation, 17, 135-152. http://dx.doi.org/10.2307/2002754

[3] Schiesser, W.S. (1991) The Numerical Methods of Lines. Academic Press, San Diego.

[4] Nakashima, M. (2001) Unconditionally Stable Explicit Difference Schemes for the Variable Coefficients Parabolic Differential Equation (II). Processing Techniques and Applications International Conference, Las Vegas, June 2001, 561-569.

[5] Nakashima, M. (2002) Unconditionally Stable Explicit Difference Schemes for the Variable Coefficients Parabolic Differential Equation (IV). In: Dinov et al., Eds., Numerical Methods and Applications, Lecture Notes in Computer Science, Springer, Vol. 2542, 536-544.

[6] Nakashima, M. (2003) Unconditionally Stable Explicit Difference Schemes for the Variable Coefficients Two Dimensional Parabolic Differential Equation (V). Journal of Applied Mechanics, 54, 327-341. 